\title{
Erratum: Polarization of M2 macrophages requires Lamtor1 that integrates cytokine and amino-acid signals
}

Tetsuya Kimura, Shigeyuki Nada, Noriko Takegahara, Tatsusada Okuno, Satoshi Nojima, Sujin Kang, Daisuke Ito, Keiko Morimoto, Takashi Hosokawa, Yoshitomo Hayama, Yuichi Mitsui, Natsuki Sakurai, Hana Sarashina-Kida, Masayuki Nishide, Yohei Maeda, Hyota Takamatsu, Daisuke Okuzaki, Masaki Yamada, Masato Okada \& Atsushi Kumanogoh

Nature Communications 7:13130 doi: 10.1038/ncomms13130 (2016); Published 12 Oct 2016; Updated 27 Feb 2017

In this Article, there are errors in the labelling of the $x$ axis in Fig. 5g,h that were introduced during the production process. The second label on the $x$ axis of each graph in Fig. $5 \mathrm{~g}$ should have been ' ${ }_{\Phi} \mathrm{WT}$ ' instead of ' ${ }_{\Phi} \mathrm{KO}$ '. In Fig. 5h, the labels ' 0 ', ' 5 ', ' 10 ' and ' 0 ' should have been ' 0 ', ' 1 ', ' 5 ' and ' 10 ', respectively. In addition, lane labels indicating the duration of leucine stimulation are missing from the Western blot in Fig. 5b, and should have read ' 0 ', ' 5 ', ' 10 ', ' 15 ', '20, ' 0 ', ' 5 ', ' 10 ', ' 15 ' and '20', from left to right. The correct version of Fig. 5 appears below as Fig. 1 . 
a

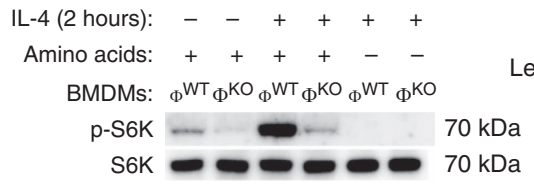

d

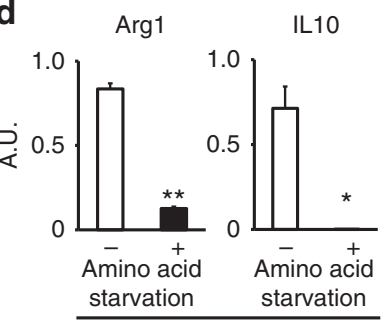

$\mathrm{IL}-4,2$ days

e b

BMDMs: $\quad \Phi^{\mathrm{WT}} \quad \Phi^{\mathrm{KO}}$

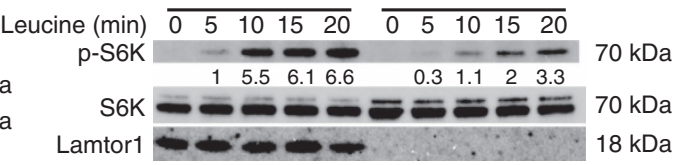

C

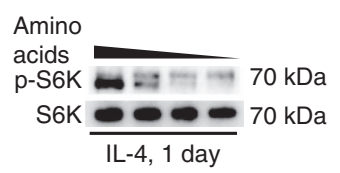

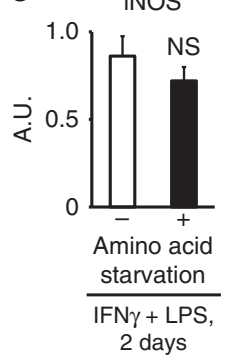

f

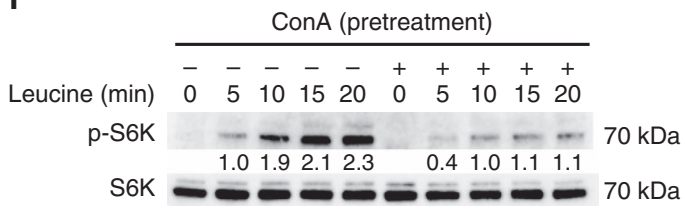

g
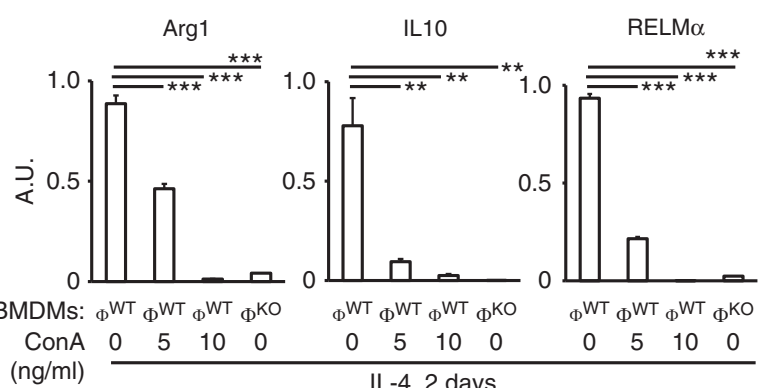

h

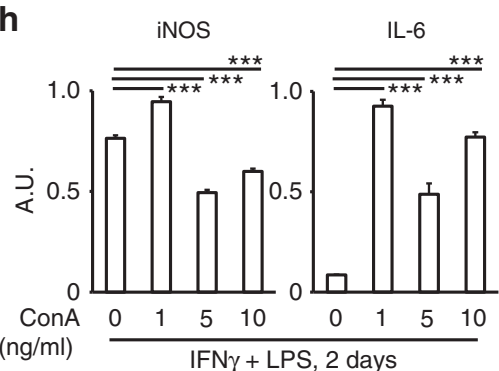

BMDMs: ${ }_{\phi}{ }^{W T}{ }_{\Phi}{ }^{W T}{ }_{\Phi}{ }^{W T}{ }_{\Phi^{K}}$

ConA $0 \quad 5 \quad 10$

$(\mathrm{ng} / \mathrm{ml})$

IL-4, 2 days

i $\quad \begin{array}{lll}\operatorname{Arg} 1 & \mathrm{IL} 10 & \mathrm{RELM} \alpha\end{array}$

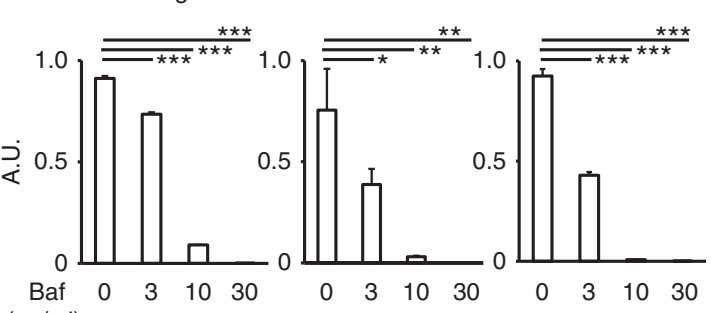
$(\mathrm{ng} / \mathrm{ml})$

IL-4, 2 days

I

Glucose $\left(\mathrm{mg} \mathrm{dl}^{-1}\right): \begin{array}{lllll}400 & 50 & 10 & 0 & 400\end{array}$

Amino acids:

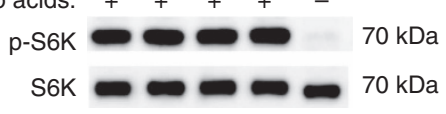

m

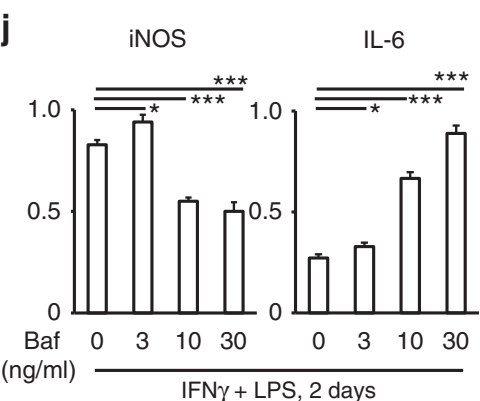

k ConA (ng/ml): $\begin{array}{lllcc}0 & 0 & 10 & 0\end{array}$ Torin1 (nM): $0 \quad 0 \quad 0 \quad 250$ p-STAT6 $=100 \mathrm{kDa}$ STAT6 $-\longrightarrow 100 \mathrm{kDa}$

Figure 1 |

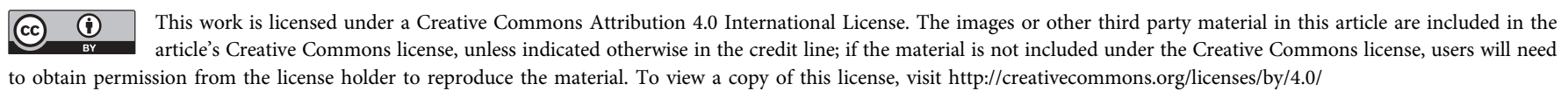

(C) The Author(s) 2017 